\title{
Corynebacterium kutscheri
}

National Cancer Institute

\section{Source}

National Cancer Institute. Corynebacterium kutscheri. NCI Thesaurus. Code C86314.

A species of aerobic or facultatively anaerobic, Gram-positive, coryneform, pleomorphic bacilli assigned to the phylum Actinobacteria. This species is nonmotile, non-spore forming, catalase positive, reduces nitrate, hydrolyzes urea and esculin, is pyrazinamidase positive, and is negative for the CAMP test. C. kutscheri is commensal in rodent species and is associated with infections from rat bites. 